\title{
Advanced Gurney With Voice Module Operation
}

\author{
B. Janardhana Rao ${ }^{1}$, Assistant Professor, O. Venkata Krishna ${ }^{2}$, Associate Professor \\ ${ }^{1}$ CVR College of Engineering College, ECE, Hyderabad, India \\ Email: janardhan.bitra@gmail.com \\ ${ }^{2}$ CVR College of Engineering, EIE, Hyderabad, India \\ Email: venkatakrishna.odugu@gmail.com
}

\begin{abstract}
Speech is the vocalized form of human communication. Speech is the most natural means of communication between people. The people with disabilities and blind are unable to convey the information so the speech recognition system is used in these cases to share the information by operating computer through voice input. The paper is based on speech recognition technology which is having more number of applications in all areas of research and is the fast growing technology. Hence lot of researchers paid their attention to develop technical means which allows distant persons to communicate with each other. The main aim of this project is to provide hand free voice communication to control the direction and the speed of an advanced gurney with voice commands. This system provides comfortable conversation by the utilization of microphone. The speech module is trained with the necessary commands that enable the navigation of the gurney. The speech module is then interfaced to ARM7 and through Zigbee module the navigation of the gurney is obtained. All the processing can be realized in real-time.
\end{abstract}

Index Terms-Speech recognition, gurney, ARM7, Zigbee.

\section{INTRODUCTION}

Speech is the most natural means of communication between people. The words spoken by the human using microphone is captured by the computer which is called speech recognition technology. The step by step process of speech recognition is discussed in the following sections. In ideal case speech recognition taught by a human but in practical case this depends on factors like multiple users, vocabulary and noise environment. Speech recognition is basically the conversion of sound or speech energy into electrical signals which are further sampled into digital signals. The interaction with the machines in the future is completely changed by using speech recognition system. In the future using this speech recognition system, the way to interact with machine will change. The scientists are working to overcome the limitations of this since it is improving day by day.

Nowadays in the field of aerospace the training and operational demands on the crew have significantly increased with the proliferation of technology is introduced by the voice recognition technology and using the simple voice commands one can control the cameras, lights, pumps and some equipment. Hence, lot of researchers are concentrating to develop a technical means which allow distinct persons to communicate in such a way as they are near each other.

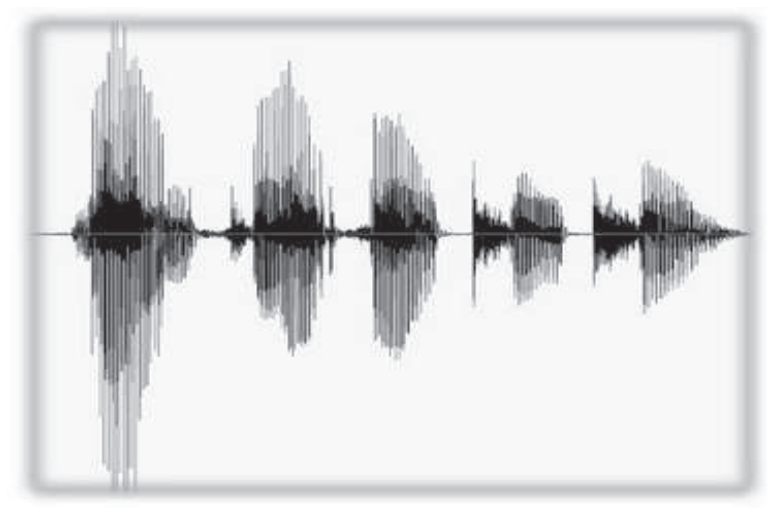

Fig 1: Speech or voice as an analog signal

\section{Speech Recognition System}

The speech recognition IC HM2007 is used for taking speech recognition [1]. It is completely assembled and easy to use programmable speech recognition circuit. The circuit is trained with the words you want the circuit to recognize is called programmable and many facets of speech recognition systems can experiment with this board. For further developments, nay microcontroller can interface with the 8 bit data output available in the board. The interfacing applications include robotics movements, controlling home appliances, speech to text translation and speech assisted technologies. The diagrammatic representation of Speech Recognition kit (SR-07) with HM2007 IC is shown in Fig 2 [2][3].

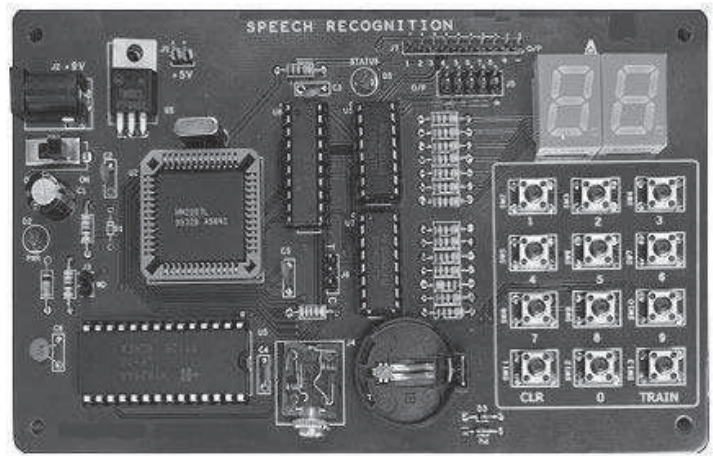

Fig 2: Speech recognition Kit 
In order to control toys, tools, home appliances and computers performed by using speech recognition system. At its basic level, speech controlled appliances and tools allow the user to perform parallel tasks (i.e hands and eyes are busy elsewhere) while working with the tools or appliances. The speech recognition IC HM2007 is the major part of the circuit. The IC can recognize 20 words, each word a length of 1.92 seconds.

\section{A) Operation of Speech Recognition}

The internal process in HM2007 IC is shown in Fig 3. The main advantage of the IC 2007 is that it provides a complete package for speech detection purpose.

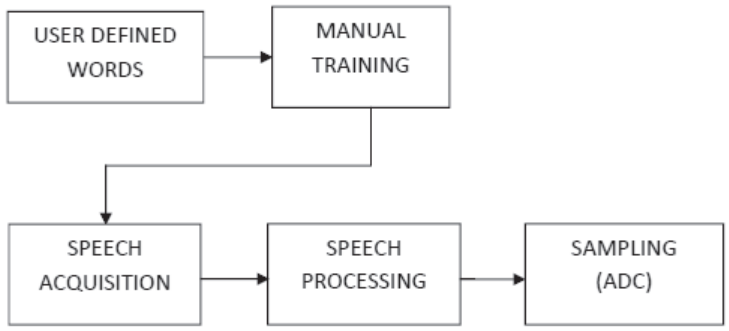

Fig 3: Internal Process in HM2007 IC

Speech Acquisition: In real time applications the speech samples are stored in memory for processing which are obtained from the speaker. The digital speech is obtained from the speech acquisition by combining the microphone and analog to digital converter with proper amplification to receive voice signal and sampling it. The system sends the analog speech through a transducer, amplifies it, and sends to ADC. The received samples of speech stored in memory on a RAM. The speech acquisition can implement easily with HM2007 IC. The audio codec receives the signal from microphone input port, amplifies it, and converts it into 8-bit PCM digital samples at a sampling rate of $3.57 \mathrm{MHz}$ [5]. The initial configurations or training of words required for HM2007 performed by using a programming board. The particular value is assigned for each word by speaking words into the microphone under the training process. For example "left can be assigned a value 01 or 04 . For further functions this can be connected to a microcontroller.

Speech Preprocessing: The speech signal consists of the uttered digit along with a pause period and background noise. The amount of processing required is reduced by using preprocessing in later stages. The preprocessing of the speech signal involves takes the input speech samples, blocking the samples into frames and returning a unique pattern for each sample, which are described in following steps

1) The significant samples of the speech signal must identified by the system. The system divides the speech samples into overlapped frames to accomplish this goal. 2) Using endpoint detection and energy threshold calculations the system checks the frames for voice activity.

3) The speech samples are passed through the preemphasis filter.

\section{Training The Speech Recognition Kit}

The microphone audio signals are analyzed by using HM2007. The HM2007 interfaces directly with a microphone and the microcontroller. The word length to be recognized will be selected as the highest amount of 1.92 seconds. The maximum of 20 words allowed with the $8 \mathrm{~K}$ byte memory used. These will suit to the needs of work, as the speech commands will never surpass 20 words. If the commands are speech commands that affects volume control determined by preceding the audio signal in HM2007. The HM2007 chip takes the input from microphone. The maximum word length is set to 1.92 seconds and a 20 word capability by adjusting the 13 th pin i.e. WLEN of the HM2007 IC to HIGH (instead of 0.96 seconds and a 40 -word capability when the $13^{\text {th }}$ pin is in LOW state). Pin 15, CPUM (CPU Mode) is set to low for manual mode of operation. This configuration used in entire work of preceding the speech signal. In order to provide better accuracy than alternate setup, the smaller word capacity is used for HM2007.

For training mode a target class index is required. A target class index is defined as; a number is pressed on the keypad representing a specific target class index i.e 0 to 1 numerical numbers. A target class index is defined as the desired out come on the display device. The maximum number of 1 to 20 class indices programmed can be anywhere. The turn-off condition of LED indicates the system is ready for training. First, the word slot 01 is spoken when the TRN button is pressed on the keypad. The LED will blinks for indicating the word has been loaded or trained. This same process is continuing for as many slots as required based on the full capacity of the system. Whenever training is completed then the LED remains illuminated. The flow chart for training the speech recognition kit is shown in Fig 4.

For testing the speech recognition process, just followed the same procedure of the training process into the microphone. The memory number of the word is displayed on the digital display, For example, if the word 1 is trained for forward direction, then saying the directory into the microphone will cause the number 01 to be displayed. Fig 5 represents the flowchart for testing mode. 


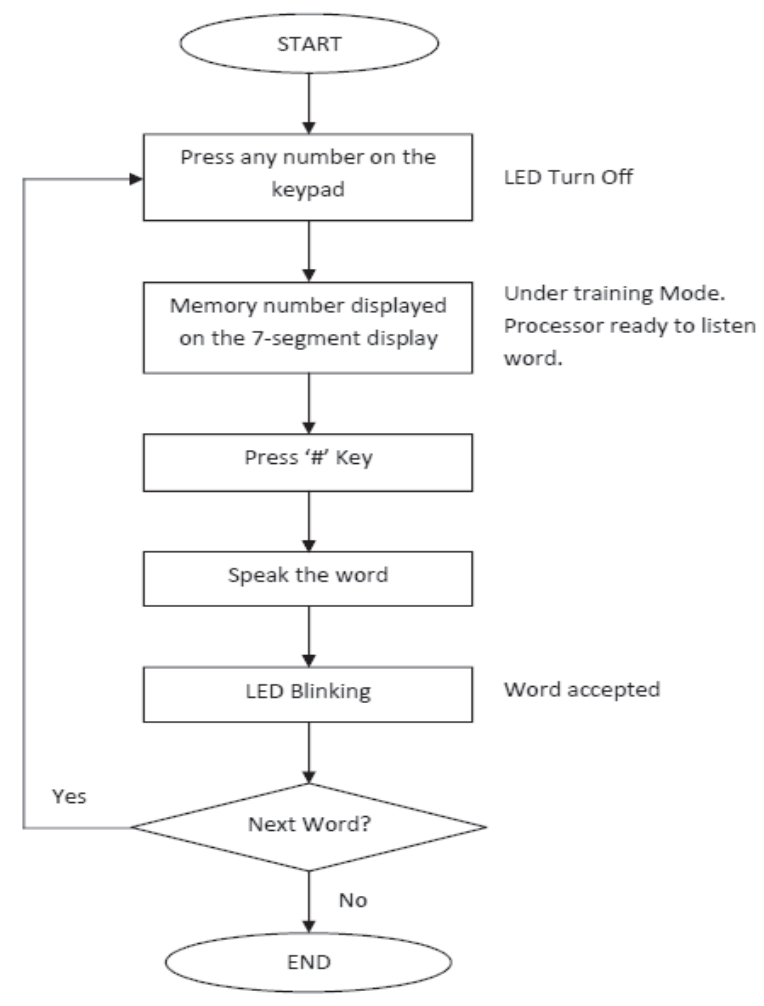

Fig 4: The flow chart for training the speech recognition kit

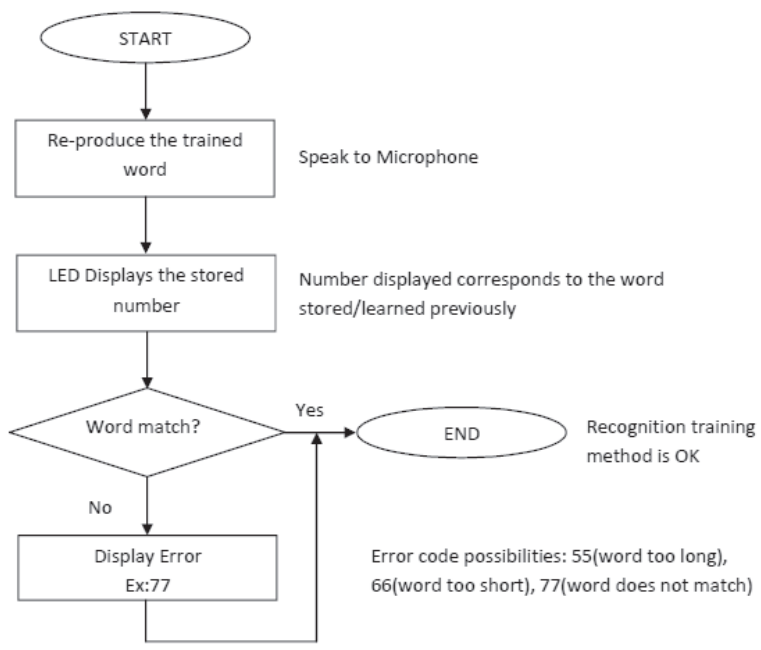

Fig 5: The flow chart for the word recognition testing mode

\section{Design And Implementation}

In this section, the implementation of modules explained in detail. First the speech recognition module receives speech commands from the MIC. The module consists of HM2007-IC for the processing of the voice and matching the received voice signal with stored voice. A SRAM is connected to the HM2007 IC to store the all voice commands. The main voice recognition module provides the decoders to decode the voice signals and the decoded signals are forwarded to the transmission microcontroller LPC2148. The LPC2148 controller processes the decoded signal and sends it to the Zigbee transceiver. The Zigbee transceiver from the control section transmits the signal to the gurney section's Zigbee Transceiver. The diagrammatic representation for the control section is shown in Fig 6.

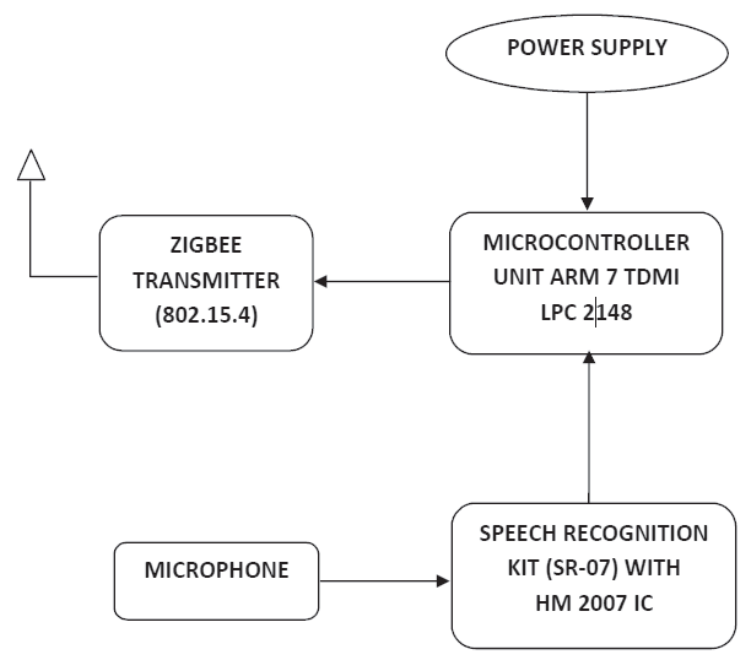

Fig 6: Block Diagram of the Transmitting Module (Control Section)

At the gurney section, the Zigbee transceiver receives the signals from the transmission module. The transmission module provides the signals to the microcontroller AT 89S52. The microcontroller decodes the signals received from the Zigbee and processes them. The processed signals are given as an input to the Motor Driver circuit L293D which is connected to the gurney. The gurney moves according signal provided by the AT89S52 microcontroller. The diagrammatic representation for the receiver section is shown in Fig 7.

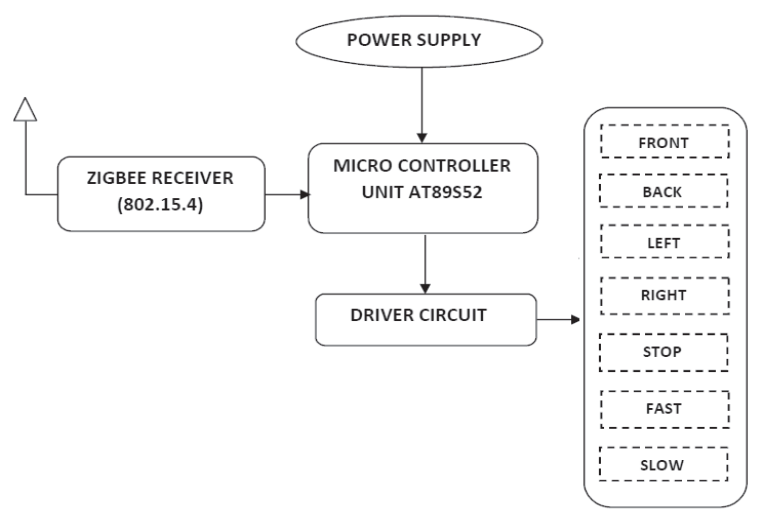

Fig 7: Block Diagram of the Receiving Module (Gurney Section)

This chapter describes the implementation of an advanced gurney which is controlled by speech commands. This type of a system is based on Speech Recognition Kit (SR-07) with Speech Recognition IC

(HM 2007) which provides speech commands as input to the ARM7 processor [4], AT889C51 microcontroller for 
controlling the gurney [6], relays for controlling and Zigbee as data transceiver. The ARM7 microcontroller is connected to Speech Recognition kit. This speech recognition kit transfers the speech signals to the ARM7 microcontroller. In this design GPIO pins interface is used to provide communication between ARM7 TDMI and the speech recognition kit. Port P0.0 is used for transmission of data i.e. speech commands from ARM7 to the Zigbee module and Port P0.1 is used for reception of speech commands from the speech recognition kit to the ARM7.

At the transmitter section LPC2148 microcontroller is used for controlling the speech recognition kit and the zigbee module, the schematic representation for transmitter section is shown in Fig 8.

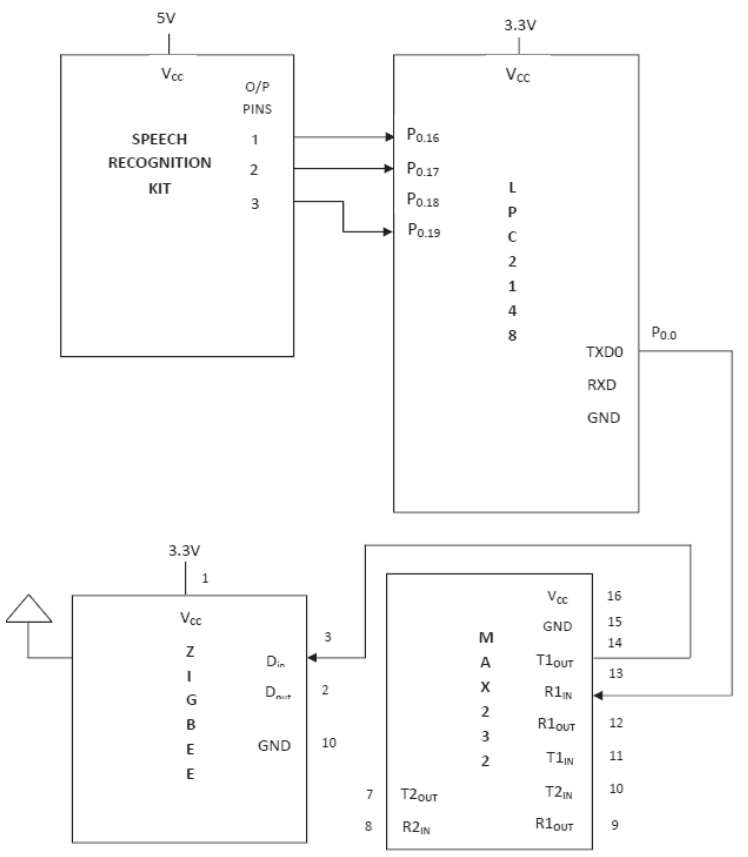

Fig 8: Schematic representation for the transmitter section

At the receiver section AT89S52 micro-controller is used for controlling the DC motor driver, Zigbee module and relay. The schematic representation for transmitter section is shown in Fig 9.
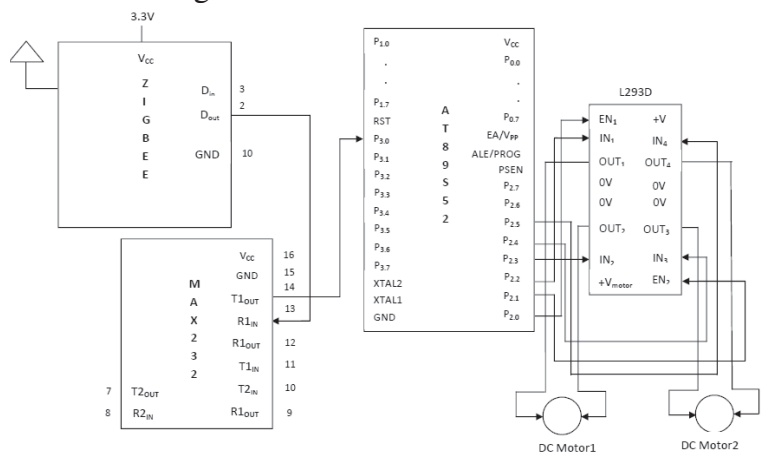

Fig 9: Schematic representation of the receiver section

\section{Experimental Results}

The speech recognition kit is trained according to the requirement. The trained commands are stored in their respective address. Finally, the expected output i.e. movement of the gurney followed according to the speech commands is obtained. Each command trained will be stored at a particular memory address and this address will be displayed on the seven-segment display which is associated with the speech recognition kit. The commands trained can also be tested by pronouncing the words which were trained. On the spell of the command the sevensegment display must show the address at which that particular command has been stored. For example, while testing, if we spell "RIGHT" the address location has to be displayed "06". The commands trained/tested and their respective memory addresses displayed on the sevensegment display are listed below with pictures:

Transmitter section containing speech recognition kit showing address location 01 is shown in the fig 10(i). The command stored at this particular address is "FRONT".

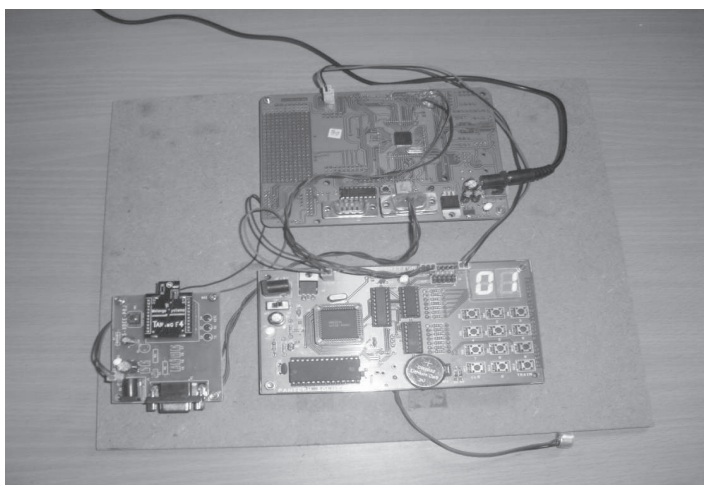

Fig 10(i): Transmitter/Control Section showing the address location 01

Transmitter section containing speech recognition kit showing address location 02 is shown in the Fig 10(ii). The command stored at this particular address is "BACK".

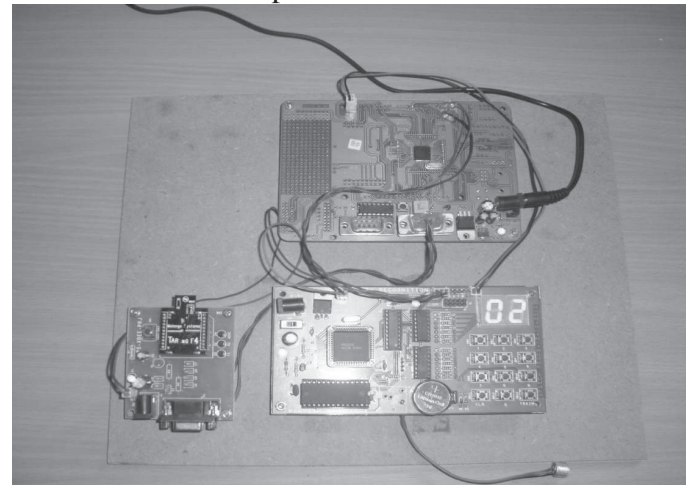

Fig 10(ii): Transmitter/Control Section showing the address location 02

Transmitter section containing speech recognition kit showing address location 03 is shown in the Fig 10(iii). The command stored at this particular address is "LEFT 


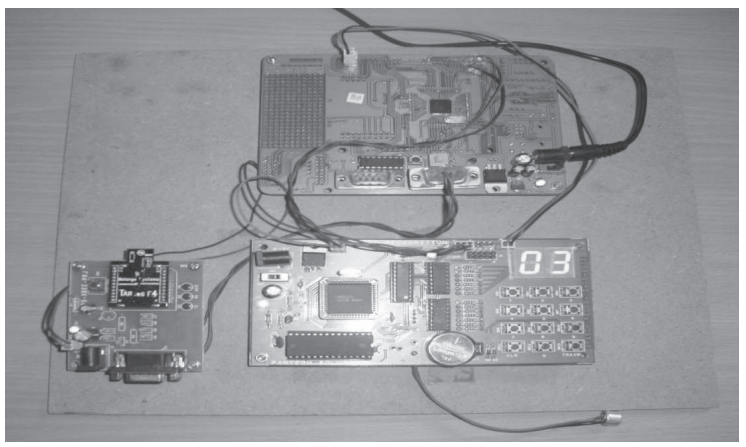

Fig 10 (iii): Transmitter/Control Section showing the address location 03

Transmitter section containing speech recognition kit showing address location 04 is shown in the figure (iv). The command stored at this particular address is "RIGHT".

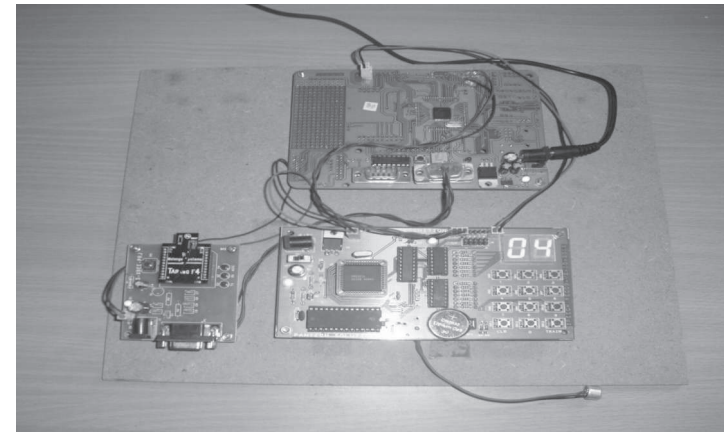

Fig 10(iv): Transmitter/Control Section showing the address location 04

Transmitter section containing speech recognition kit showing address location 05 is shown in the figure (v). The command stored at this particular address is "STOP".

Transmitter section containing speech recognition kit showing address location 06 is shown in the Fig 10(vi). The command stored at this particular address is "FAST".

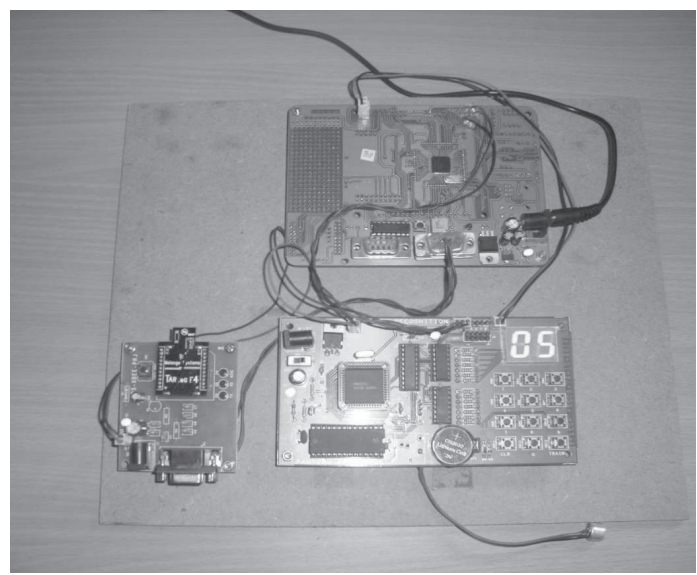

Fig 10(v): Transmitter/Control Section showing the address location 05

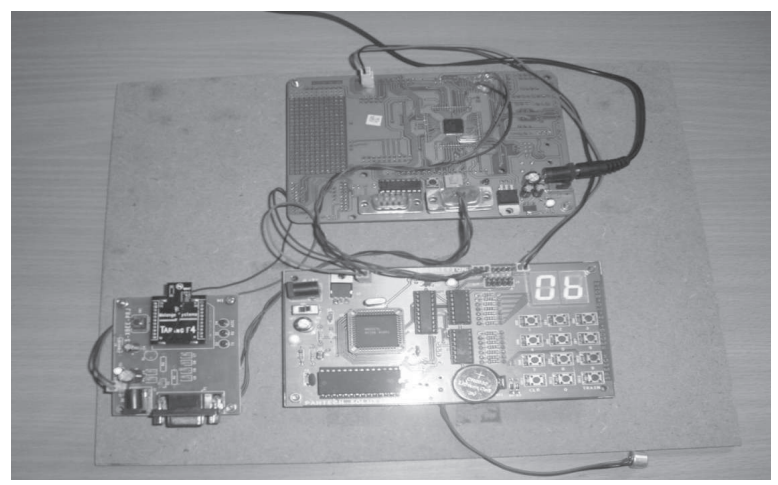

Fig 10(vi): Transmitter/Control Section showing the address location 06

Transmitter section containing speech recognition kit showing address location 07 is shown in the Fig 10(vii). The command stored at this particular address is "SLOW".

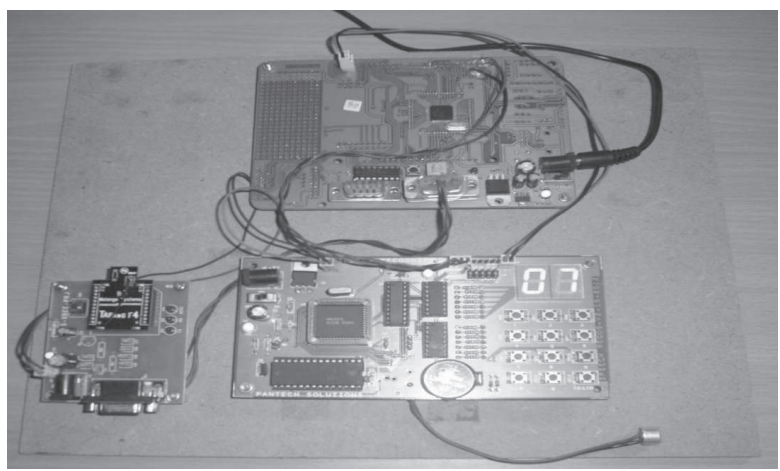

Fig 10(vii): Transmitter/ Control Section showing the address location 07

Gurney/Receiver section is controlled by the transmitter section containing the speech recognition kit. The gurney is controlled by the speech/voice commands trained. The gurney section which navigates according to the speech commands is shown in Fig 10(viii).

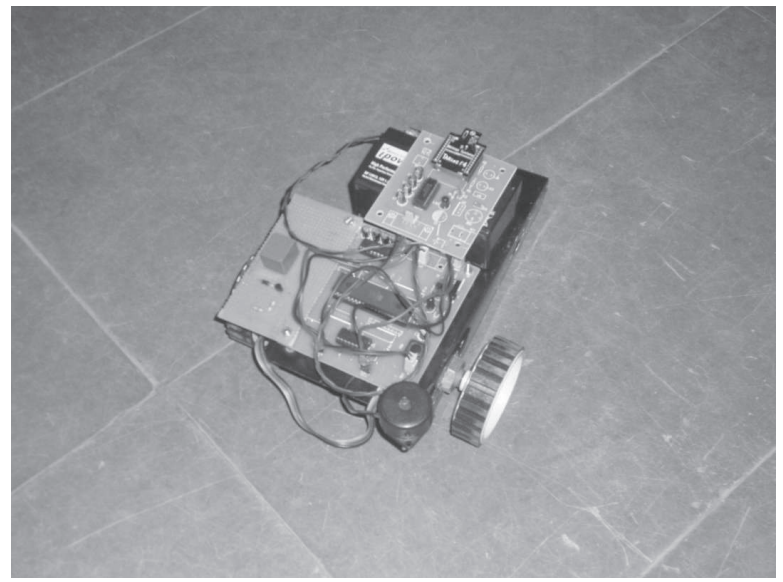

Fig 10 (viii): Receiver/Gurney Section controlled by speech/voice commands. 


\section{Conclusion}

In this paper an intelligent gurney which is controlled by speech has been developed. The user can train the required commands by the utilization of a microphone. The user trained commands are used to control the navigation path of the gurney and to which the gurney navigates accordingly. Not only the direction but also the speed of the gurney can be controlled. The accuracy of the speech commands received by the gurney section is maximum. The speech commands trained by the user are assigned their particular address locations during the time of coding itself. If any individual wants to change the address locations, the change should be done in the code itself. The drawbacks of the system are noisy environment. The background noise may corrupt the original information or data under noisy environment.

\section{REFERENCES}

[6] Sudheer kanuri, T V Janardhana Rao, Ch Sridevi, M S Madhan Mohan "Voice and Gesture Based ElectricPowered Wheelchair Using ARM" IJRCCT, Vol-1, Issue 6, pp 278-283.

[7] Preeti Saini, Parneet Kaur. "Automatic Speech Recognition: A Review". International Journal of Engineering Trends and Technology (IJETT). V4(2):132-136 Feb 2013. ISSN:22315381

[8] M.A.Anusuya and S.K.Katti , “ Speech Recognition by Machine: A Review ", (IJCSIS) International Journal of Computer Science and Information Security, vol. 6, no. 3, pp. 181 - 205, 2009.

[9] P.Vishnu Kumar, Dr.T.JayaChandra Prasad"The Design Of Speech-Control Power Point Presentation Tool Using Arm7"International Journal of Computer Trends and Technology (IJCTT),V3(4):459-463 Issue 2012 .ISSN 22312803.

[10] Yahya S. H. Khraisat "Speaker Independent Voice Recognition Calculator" Contemporary Engineering Sciences, Vol. 5, 2012, no. 3, $119-125$.

[11] Sunpreet Kaur Nanda, Akshay P.Dhande "Microcontroller Implementation of a Voice Command Recognition System for Human Machine Interface in Embedded System" International Journal of Electronics, Communication \& Soft Computing Science and Engineering (IJECSCSE) Volume 1, Issue 1, pp 5-8. 